\title{
SOILomics; where Microbiome Genetics meets Precision Agriculture
}

\author{
Dimitrios Vlachakis ${ }^{1,2,3}$, Aspasia Efthimiadou ${ }^{\square}$ \\ ${ }^{1}$ Laboratory of Genetics, Department of Biotechnology, School of Applied Biology and Biotechnology, Agricultural University of \\ Athens, Greece \\ ${ }^{2}$ Lab of Molecular Endocrinology, Center of Clinical, Experimental Surgery and Translational Research, Biomedical Research \\ Foundation of the Academy of Athens, Greece \\ ${ }^{3}$ Department of Informatics, Faculty of Natural and Mathematical Sciences, King's College London, Strand, London WC2R 2LS, \\ United Kingdom \\ ${ }^{4}$ Hellenic Agricultural Organization-Demeter, Institute of Soil and Water Resources, Department of Soil Science of Athens, Lycovrisi, \\ Greece
}

Competing interests: DV none; AE none

\section{Abstract}

Advances in genetics, soil biochemistry and microbiome analysis are opening up a new era in Precision Agriculture. In this direction, new techniques bring groundbreaking changes in land management practices through direct or indirect management of soil microbial communities. There is huge demand for the protection and enhancement of soil health and climate change resilience of crops. The increase in population, food consumption and fast approaching climate change pose a new threat to mankind that only by being proactive and highly prepared to deploy all novel and innovative stratagems in state-of-the-art soil microbiome precision agriculture can be avoided.

\section{Introduction}

Agriculture is one of the most exposed sectors to increased climate variability. Soil degradation, loss of biodiversity, increased air and water pollution affect the viability of the crop and the agricultural productivity. Nowadays, new and pioneering strategies utilizing the genetic flexibility and biochemical power of the microbes are being formulated. Those approaches attempt to coordinate and thus transform the microbial content of the field into a natural molecular plant to optimize and shield the crop and at the same time protect the environment mainly by reducing chemical interventions. In this direction, multidimensional approaches combining science and know-how from different fields aim for the capture of soil microbiome using homogeneous technologies, the traditional recording of soil and atmospheric conditions, and the creation of intelligent systems and algorithms for the management of resources and inputs based on microbiome analysis within a sophisticated Precision Agriculture System. The methodology that is being developed is primarily based on a very economical instrument, the lysimeter, which can be placed in the field easily and at low cost, and through an initial dense sampling of soil and organic technologies, the microbial profile of the crop will be attributed. The creation and establishment of a decision support system will minimize the required quantity of samples and at a lower cost will ensure the maximum reliability of the method. At the same time, the correlation of the results of the microbial analysis with the corresponding conditions and yields of crops through machine learning techniques will lead us to a sustainable approach of the agricultural systems.

Furthermore, major changes in the environment and socio-economic factors, combined with the steady increase in the earth's population, are impeding the food industry. The importance of soil microbiome in establishing the stability of the ecosystem along with its high involvement in sustainable agriculture is recognized in the past decade. Soil microbiome affects crop growth and soil functions, especially biological soil activity and fertility. Soil microorganisms contribute to soil health by cycling nutrients (such as nitrogen and phosphate) essential for plant growth and global biogeochemical cycles, they improve soil structure by increasing organic matter content, enhance the resilience of the plants by responding to environmental stresses, and confer disease resistance to crops by out-competing pathogenic microbes. Exploiting and modulating the metabolic capabilities of the soil microbiome by

\section{Article history \\ Received: 14 August 2020 \\ Accepted: 05 October 2020 \\ Published: 20 October 2021}

( 2021 Vlachakis et al.; the authors have retained copyright and granted the Journal right of first publication; the work has been simultaneously released under a Creative Commons Attribution Licence, which allows others to share the work, while acknowledging the original authorship and initial publication in this Journal. The full licence notice is available at http://journal.embnet.org. 
genetically manipulating the beneficial native microbes that promote plant health and quality are ultimate practices for achieving stable yield and reduced impact on the agroecosystem. On a different note, microbiome members may also be associated with diseases and pathogenic effects on plants. The application of pesticides and agrochemicals against phytopathogens in intensive farming leads to impairment of soil functions and long-term crop yield losses, along with negative effects on beneficial soil microbiota. Thus, the management of the microbial communities and the study of their diversity, connectivity and impact on the soil health is a key component to maintain agricultural productivity and protect the environment. Climate change favors the spread of new or migratory microorganisms and alters the microbiome distribution in the soil, affecting not only its relative abundance, but also its function. Moreover, global warming directly changes respiration rates of soil microorganisms as the processes they mediate are temperature sensitive. Soil microorganisms are mainly responsible for cycling of soil organic carbon and other nutrients, and are key players in climate feedback through the production or consumption of greenhouse gases such as $\mathrm{CO}_{2}, \mathrm{CH}_{4}$ and $\mathrm{N}_{2} \mathrm{O}$. A better understanding of soil microbiome connectivity, physiology and metabolism and how it is impacted by climate change is required for sustainable soil management.

All in all, the advances in genetics, soil biochemistry and microbiome analysis are opening up a new era in precision agriculture. In this direction new techniques push to bring groundbreaking changes in land management practices through direct or indirect manipulation of soil microbial communities. There is huge demand and great need for the protection and enhancement of soil health and climate change resilience of crops. The increase in population, consumption of foods and fast approaching climate change pose a new threat to mankind that only by being proactive and highly prepared to deploy all novel and innovative stratagems in state-of-the-art soil microbiome precision agriculture can be avoided. 